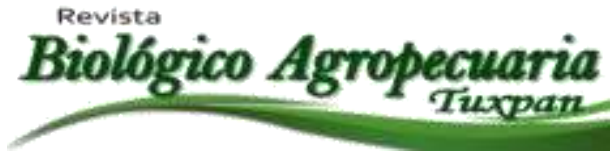

\title{
Control de la mosca pinta de los pastos Aenolamia spp con tres cepas del hongo Metarhizium anisopliae
}

Fly pint Control Aenolamia spp pasture with three strains of the fungus Metarhizium anisopliae León Hernández Miguel ${ }^{1}$, Julio César González Cárdenas ${ }^{2}{ }^{凶}$, Alfredo González Acosta $^{2}$, Alfredo González Castro $^{2}$ y José Luís Alanís Méndez ${ }^{2}$

${ }^{1}$ Estudiante de la Maestría. Facultad de Ciencias Biológicas y Agropecuarias. 2 Académico de la Facultad de Ciencias Biológicas y Agropecuaria. Universidad Veracruzana. Carretera Tuxpan-Tampico Km. 7.5. Tuxpan de R. Cano, Veracruz.

${ }^{\bowtie}$ Autor para correspondencia: juliogonzalez@uv.mx. .

Recibido: 14/01/2014

Aceptado: 18/07/2014

\section{RESUMEN}

Se evaluaron tres cepas del hongo Metarhizium anisopliae y un control, dos de ellas se aislaron de dos productos comerciales: Specific y Meta-Sin ambas con concentración de conidios de 2.5 x $10^{12}$ esporas y en presentación en polvo, la tercera cepa fue de un aislamiento original de mosca pinta (Aenolamia sp.) colectada de adultos parasitados y aislado en un medio de a base de papa-dextrosa-agar (PDA) y reproducida en arroz como medio sólido denominada como Meta 1, y el control que fue agua la utilizada, la forma de aplicación fue de aspersión utilizando una aspersora de capacidad de 15 litros, la dosis del hongo fue de $2.5 \times 10^{12}$ esporas/ha para el tratamiento. Se realizaron cinco aplicaciones con intervalos de 15 días, los muestreos se realizaron con frecuencia de 8 días. Los tratamientos evaluados quedaron de la siguiente manera: Tratamiento 1. Meta 1. $2.5 \times 10^{12}$ esporas/ha, Tratamiento 2. Specific $2.5 \times 10^{12}$ esporas/ha, Tratamiento 3. Meta-Sin $2.5 \times 10^{12}$ esporas/ha, Tratamiento 4. Control (Agua). Se realizaron cuatro repeticiones de cada uno de los tratamientos, teniendo un total de 16 parcelas. Se utilizó un diseño de bloques completamente al azar. Las variables de respuesta a evaluar fue la población de las ninfas y adultos, antes y después de la aplicación. Se realizaron nueve muestreos en intervalos de ocho días durante un periodo de 3 meses utilizando un marco metálico de $1 \mathrm{~m}$ x $1 \mathrm{~m}$ para el caso de ninfas y para adultos se utilizó una red entomológica. Existieron diferencias significativas (P> 0.05) entre los tratamientos evaluados hasta el cuarto muestreo tanto en ninfas como adultos, siendo el mejor el tratamiento 1Meta, seguido por Specific 2.5 x $10^{12}$ esporas/ha y tercero el Tratamiento 3. Meta-Sin $2.5 \times 10^{12}$ esporas/ha comparado con el testigo control.

\section{Revista Científica Biológico Agropecuaria Tuxpan 2 (1)}




\begin{abstract}
We evaluated three strains of the fungus Metarhizium anisopliae and control, two of which were isolated from two commercial products: Specific and Meta-Without both with concentration of $2.5 \mathrm{x}$ 1012 conidia spores and in powdered form, the third strain was an isolate spittlebug original (Aenolamia sp.) adults collected from parasitized and isolated in a medium based on potato dextrose agar (PDA) and reproduced in rice as a solid medium known as Target 1, and water control was used , the shape of the spray application was spray using a 15-liter capacity, the dose of the fungus was $2.5 \mathrm{x}$ 1012 spores / ha for treatment. Five applications were made at intervals of 15 days, the samples were performed every 8 days. The treatments were as follows: Treatment 1 . Goal 1. $2.5 \times 1012$ spores / ha, Treatment 2. Specific 2.5 x 1012 spores / ha, Treatment 3. Meta-Sin 2.5 x 1012 spores / ha, Treatment

4. Control (water). There were four replicates of each treatment, with a total of 16 plots. Design was a randomized complete block. The response variables were evaluated population of adults and nymphs before and after application. Nine samples were performed at intervals of eight days over a period of 3 months using a metal frame of $1 \mathrm{~m} \times 1 \mathrm{~m}$ in the case of nymphs and adults used a sweep net. Significant difference $(\mathrm{P}>0.05)$ between treatments until the fourth sampling both nymphs and adults, being the best 1Meta treatment, followed by Specific 2.5 x 1012 spores / ha and third Treatment 3. Meta-Sin 2.5 x 1012 spores / ha compared with the control control.
\end{abstract}

\section{INTRODUCCIÓN}

La superficie territorial del estado de Veracruz es de 7’000,281.5 hectáreas, de los que 3'000,687.4 se dedican a la ganadería; es decir, 50.6\% del total (INEGI, 2012). Concretamente, la ganadería bovina ha sido y es una de las actividades más dinámicas, y se considera que su práctica repercute sobre diversos aspectos de la sociedad; aunado a lo anterior, se le considera un factor fundamental en la constitución de los complejos sistemas ecológicos que definen la singular biodiversidad del estado (Hernández et al. 2006).

En el estado de Veracruz, la ganadería de pastoreo representa la principal actividad pecuaria en más de 50\% de su territorio y tiene una gran importancia social, cultural y ambiental.
Entre las especies de gramíneas más difundidas durante las décadas más recientes destacan las del género Brachiaria, que es una especie de alta capacidad para establecerse en suelos ácidos de baja fertilidad, y de fácil propagación a través de semillas o material vegetativo y además produce cantidades aceptables de forraje de buena calidad. Sin embargo, una de sus limitaciones es la susceptibilidad al ataque del salivazo (Homoptera: Cercopidae) (Lapointe, 1993; Rivas y Holmann, 2004).

La presencia de la plaga en los pastizales está estrechamente relacionada con el patrón de lluvias y puede tener hasta seis generaciones dependiendo del periodo lluvioso. Con un ataque severo de salivazo, la parte aérea de la planta muere $y$ se reduce significativamente la producción de materia seca, así como la digestibilidad y la calidad del forraje, con un impacto negativo sobre la carga

\title{
Revista Científica Biológico Agropecuaria Tuxpan 2 (1)
} ISSN: 2007-6940 
animal y la producción de leche y carne (Holman y Peck, 2002).

Para el control de esta plaga se han utilizado por mucho tempo insecticidas de varios grupos toxicológicos teniendo como consecuencia efectos negativos en el suelo, contaminación del agua, medio ambiente, y que en poco tiempo podrían mencionarse la resistencia de esta plaga. Una solución a este tipo de problema es la introducción de programas de control de plagas con organismos benéficos $\mathrm{u}$ hongos entomopatógenos como es el caso de Metarhizium anisopliae. En la base a los resultados obtenidos en los últimos tiempos, el uso de Metarhizium anisopliae en la caña de azúcar y en los pastizales se ha expandido en Venezuela y Costa Rica, lo que ha reducido al mínimo la aplicación de productos químicos. Además, M. anisopliae no es dañino para la salud (Marrufo, 1973). Considerando estos principios, el objetivo del presente trabajo fue evaluar la efectividad del hongo Metarhizium anisopliae en el control de la mosca pinta Aenolamia sp. en el pasto Brachiaria brizanthla en la región de Tecolutla, Veracruz partiendo de una regional.

\section{MATERIALES Y MÉTODOS}

El experimento se llevó a cabo en la unidad de producción "La Escondida" ubicada en el ejido El Fuerte de Anaya del municipio de Tecolutla, Veracruz, ubicado en la zona norte del Estado, tiene una distancia de 105 kilómetros a la capital del estado. Limita al norte con el municipio de Papantla, al este con el Golfo de México, al sur con Martínez de la Torre y al Oeste limita con los municipios de Gutiérrez Zamora y Papantla. Se encuentra entre las coordenadas $20^{\circ} 29^{\prime}$ latitud norte y $97^{\circ}$ 00' longitud oeste, se ubica a una altitud promedio de 10 metros sobre el nivel del mar. Con un clima trópico-húmedo, temperatura media anual de $23.6^{\circ} \mathrm{C}$ y precipitación pluvial media anual es de 1,494 mm. La unidad de producción se encuentra establecida con pasto Brachiariala, decumbens, esta finca tenía antecedentes de haber sido atacado por mosca pinta y el control que se había utilizado había sido el químico desde su formación hasta la fecha.

Se realizaron parcelas con una superficie de $20 \mathrm{~m}$ x $20 \mathrm{~m}$ que sería las unidades experimentales, se separaron entre sí con una distancia de 10 metros, las delimitaciones se realizaron con estacas de madera

Las cepas del hongo Metarhizium anisopliae utilizados en el presente trabajo fueron las siguientes: Meta 1 denominado así a una cepa regional obtenida de moscas pintas parasitadas en el ciclo de evaluación, esta cepa primeramente fue aislada en el laboratorio de control biológico en un medio de cultivo de papa dextrosa agar, después de 12 días de esporulación, se inoculó a adultos de mosca pinta en una jaula entomológica de 40 x 40 x 50 utilizando el pasto brizanta como alimento, una vez que se observó adultos parasitados, se realizó el reaislamiento, obteniendo la cepa y realizó la reproducción del mismo en un medio sólido utilizando el arroz para ello, otra de la cepa de Metarhizium anisopliae utilizada fue de un producto comercial de nombre Specific con una concentración de conidios de $2.5 \times 10^{12}$ esporas y la otra cepa comercial de $M$. anisopliae fue obtenida por el producto MetaSin con una concentración de $2.5 \times 10^{12}$ ambas en presentación en polvo. La dosis del hongo utilizado fue de $2.5 \times 10^{12}$ esporas/ha. La aplicación se realizó con una aspersora de 15 litros de capacidad. Se realizaron cinco aplicaciones con intervalos de 15 días, los muestreos se realizaron con frecuencia de ocho días. La primera aplicación se realizó el 03 de septiembre de 2012 y la última fue el 05 de noviembre de 2012.

\section{Revista Científica Biológico Agropecuaria Tuxpan 2 (1)} ISSN: 2007-6940 
Los tratamientos evaluados quedaron de la siguiente manera: Tratamiento 1. Meta 1. 2.5 x $10^{12}$ esporas/ha, Tratamiento 2. Specific 2.5

x $10^{12}$ esporas/ha, Tratamiento 3. Meta-Sin 2.5

X $10^{12}$ esporas/ha, Tratamiento 4. Control (Agua). Se realizaron cuatro repeticiones de cada uno de los tratamientos, teniendo un total de 16 parcelas a evaluarlas cuales fueron las unidades experimentales. Se utilizó un diseño de bloques completamente al azar.

Las variables de respuesta a evaluar fue la población de las ninfas de la mosca pinta, para ello se realizó un muestreo utilizando un cuadrante de $1 \mathrm{~m}^{2}$ que se lanzaba de manera aleatoria en cuatro ocasiones en cada una de las unidades experimentales. Se realizaron nueve muestreos en intervalos de ocho días durante un periodo de 3 meses. Otra de las variables a medir fue la población de los adultos de la mosca pinta, para ello se realizó utilizando una red entomológica en cada una de las unidades experimentales se realizó el paso de 20 barridas en el centro de cada unidad experimental haciendo el conteo de adultos capturados por cada uno de las repeticiones.

\section{RESULTADOS Y DISCUSIÓN}

Se realizaron nueve muestreos en intervalos de ocho días durante un periodo de 3 meses utilizando un marco metálico de $1 \mathrm{~m}$ x 1 $\mathrm{m}$ para el caso de ninfas y para adultos se utilizó una red entomológicas. Para el caso de las ninfas el análisis de varianza mostró diferencias significativas entre las cepas (P> $0.05)$ hasta el cuarto muestreo con relación al control (testigo), mostrando una disminución de las ninfas a partir del cuarto muestreo. Esta disminución lo atribuimos a los factores climáticos que se presentaron como la baja temperatura. Los resultados obtenidos con la concentración utilizada del $2.5 \times 10^{12}$ conidios/ha concuerdan con los obtenidos por Bautista y González (2005) y Louireiro (2004) quienes estudiaron varias concentraciones del hongo Metarhizium anisopliae y observaron que a una concentración que iba de $2.5 \times 10^{10}$ a una concentración de $10^{13}$ mostraron un mejor control para las ninfas de Aenolamia sp. pero en el cultivo de la caña de azúcar. Sin embargo, Alves (1998) mostró que existe un mejor control de ninfas de salivazo cuando se realizan aspersiones de $\mathrm{M}$. anisopliae en dosis entre $5 \mathrm{x}$ 1011 y 1 x 1012 conidias/ha. Batista Filho et $a l ., 2003$ mencionan que para ejercer un mejor control de las ninfas de la mosca pinta, el hongo debe de aplicarse con un coadyuvante que permita romper la tensión superficial de la saliva del insecto y un volumen de aplicación entre los 300 y 400 1/ha. Almeida et al. (2003), mencionan que para ejercer un buen control de las ninfas, es recomendable dirigir la aspersión hacia la base de la planta, de preferencia después de las 16:00 horas, para evitar la incidencia de rayos ultravioleta.

Para conocer la efectividad de las cepas del hongo $M$. anisopliae sobre el control de los adultos, el análisis de varianza mostró diferencias significativas entre las cepas ( $\mathrm{P}>$ 0.05 ) hasta el cuarto muestreo con relación al control (testigo). Los resultados obtenidos indican que se presentó una disminución de adultos en el mes de octubre, relación estrecha a la presencia de condiciones ambientales como temperatura media de $25{ }^{\circ} \mathrm{C}$ y una humedad relativa mayor a los $80 \%$ y una precipitación pluvial mayor a los $2000 \mathrm{~mm}$, condiciones propicias a la germinación del hongos $M$. anisopliae tal como lo menciona Sosa-Gómez y Alves (2000). El tratamiento que mejor resultado se presentó fue el Tratamiento

1. Meta, que consistió en una regional de M. anisopliae, los resultados obtenidos coinciden con los encontrados por Allard et al. (1990), donde ellos aplicaron una cepa nativa de $M$. anisopliae en Trinidad y Tobago, para el control de Aeneolamia spp. Encontraron una alta proporción de adultos infectados que en aquellos que no se habían aplicado. Por otra parte, Ali (1998) en el mismo país, encontró

\section{Revista Científica Biológico Agropecuaria Tuxpan 2 (1) ISSN: 2007-6940}


que el hongo $M$. anisopliae afectó la fecundidad de Aenolamia varia saccharina, reduciendo la oviposición en un $51 \%$ en adultos expuestos al hongo.

El uso del hongo entomopatógeno $M$. anisopliae presentó una efectividad en campo en el control de la mosca pinta Aenolamia spp , este tipo de control resulta una alternativa potencial para los ganaderos de la región, ya que no contaminan el ambiente y contribuye a la inocuidad química delos alimentos (BautistaGálvez y González-Cortes, 2005). En Brasil se ha observado que el hongo M. anisopliae puede tener efectos colaterales al infectar otras plagas y que además este hongo no tiene efectos sobre algunos enemigos naturales del salivazo como son la mosca negra, Salpingogaster nigra (Schiner) y la hormiga depredadora Pheidole genalis (Borgmeier) (Mendonca y Mendonca, 2005)

\section{CONCLUSIONES}

El control biológico por el hongo $M$. anisopliae es una alternativa de control eficiente para el control de la mosca pinta de los pastos.

Buscar cepas regionales del hongo $M$. anisopliae y reproducirlas para su aplicación nos genera un buen control de la mosca pinta.

Aplicar el hongo $M$. anisopliae cuando se tengan condiciones de temperatura de $25{ }^{\circ} \mathrm{C}$ y una humedad relativa mayor a los $80 \%$ y una precipitación pluvial mayor a los $2000 \mathrm{~mm}$, ya que son condiciones propicias a la germinación del hongo.

Los hongos entomopatógenos rompen el ciclo biológico de la mosca pinta desde sus estadios iniciales (huevos y ninfas), permite disminuir las densidades de adultos de salivazo y por lo tanto las oviposiciones de los mismos.

\section{LITERATURA CITADA}

Ali, B. S. 1998. Effect of Metarhizium anisopliae var anisopliae Metschnikoff (Sorokin)

(Deuteromycotina: Hyphomycetes) on fecundity of the sugarcane froghopper, Aeneolamia varia saccharina Distant (Homoptera: Cercopidae) in Trinidad. Caroni Research and Development Division. Report, 11 p. https://doi.org/10.1016/0022-2011(90)90030-a

Allard, G. B., Chase, C. A., Heale, J. B., Isaac, J. E. y Prior, C. 1990. Field evaluation of Metarhizium anisopliae (Deuteromycotina: Hyphomycetes) as a mycoinsecticide for control of sugarcane froghopper, Aeneolamia varia saccharina (Hemiptera: Cercopidae). Journal of Invertebrate Pathology, 55 (1): 41-46. https://doi.org/10.1016/0022-2011(90)90030-a

Almeida, J. E. M., Batista Filho, A. y Santos, A. S. 2003. Avaliação do controle biológico de Mahanarva fimbriolata (Hemiptera: Cercopidae) com o fungo Metarhizium anisopliae em variedades de cana-de-açúcar e diferentes épocas de corte. Arquivos do Instituto Biologico, 70(1): 101-103.

https://doi.org/10.11606/t.11.2005.tde-29042005-165644

Alves, S. B. 1998. Fungos entomopatogênicos. In: Alves S. B. (Ed.), Controle microbiano de insetos. Piracicaba: Ed. FEALQ, 1998. 11: 289-381.

Batista Filho, A., Almeida, J. E. M., Santos, A. S., Machado, L. A. y Alves, S. B. 2003. Eficiência de isolados de Metarhizium anisopliae no controle de cigarrinha-daraiz-dacana- de-açúcar, Mahanarva fimbriolata (Hom.: Cercopidae). Arquivos do Instituto Biologico, 70(3): 309-314. https://doi.org/10.1590/s1808-16572012000100007 
Bautista- Gálvez, A. y González-Cortes, N. 2005. Tres dosis de Metarhizium anisopliae sobre la Mosca Pinta (Aeneolamia spp.) en Caña de Azúcar en la Región de los ríos, Estado de Tabasco. Universidad y Ciencia, 21(41): 37-40. https://doi.org/10.2307/j.ctv346nbm.7

Hernández, A., Salinas, V. M., Mayra L., García, R., Patricia C., Odalys U. y Álvarez, J. L. 2006. Comportamiento de las variantes genéticas de los genes қCaseína, $\alpha$-Lactoalbúmina y Hormona del crecimiento en la raza Criollo Lechero Tropical en México y su relación con el nivel de respuesta al calor, Revista de Salud Animal, 28(2): 96-104. https://doi.org/10.20318/recs.2020.5166

Holmann, F. y Peck, D. 2002. Economic damage caused by spittlebugs (Homoptera: Cercopidae) in Colombia: a first approximation of impact on animal production in Brachiaria decumbens pasatures. Neotropical Entomology, 31(2): 1-10. https://doi.org/10.1590/s1519-566x2002000200016

Instituto Nacional de Estadística, Geografía e Informática (INEGI). 2012. Censo Agrícola Ganadero. En: http://www.inegi.org.mx/est/contenidos/ espanol/metodologias/censos/sm_agrico la.pdf. Consultado el 13 de noviembre 2012.

Lapointe, S. L. 1993. Manejo de dos plagas clave para forrajes de las sabanas neotropicales. Pasturas Tropicales, 15(3):1-9.
Loureiro, E. 2004. Seleção e avaliação de campo de isolados de Metarhizium anisopliae (Metsch.) Sorok., para o controle da cigarrinha - da raiz da canade-açúcar, Mahanarva fimbriolata (Stal, 1854) (Hemiptera: Cercopidae). Universidade Estadual Paulista "Júlio de Mesquita Filho". Faculda de de Ciências Agronômicas. Campus de Botucatu, 101

p. https://doi.org/10.1590/s1808-16572012000100007

Marrufo-Espejo, R. C. 1973. Cultivo de hongo entomofágo Metarhizium anisopliae (Metch) Sor. y ensayos preliminares de su efecto contra el complejo mosca pinta de los pastos y otros insectos. Tesis IAZ. Monterrey, N.L. ITESM, 79 p.

Mendonca, A. F. y Mendonca, I. C. B. R. 2005. Cigarrinhada-raíz Mahanarva fimbriolata (Hemiptera: Cercopidae). Pp. 95-140. En: Mendonza A.F. (Ed). Cigarrinha da cana de acuzúcar: controle biológico, Insecta, Maceió. 317p. https://doi.org/10.11606/t.11.2006.tde-15012007-152427

Rivas, L. y Holmann, F. 2004. Impacto de la adopción de híbridos de Brachiaria resistentes al salivazo Colombia, México y Centroamérica. Cali, Colombia. CIAT/ILRI. 29 p.

Sosa-Gómez, D. R. y Alves, S. B. 2000. Temperature and relative humidity requirements for conidiogenesis of Bauveria bassiana (Deuteromycetes: Moniliaceae). Anais da Sociedade Entomologica do Brasil, 29(3): 515-521. https://doi.org/10.1590/s0301-80592000000300014 
Copyright (c) 2014Miguel León Hemández, Julio César Gonzâlez Cárdenas, Alfredo González Acosta, Alfredo González Castro y José Luis Alanis Méndez

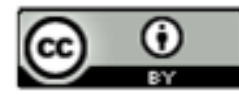

Este texto está protegido por una licencia Creative Common 4.0 .

Usted es libre para Compartir —copiar y redistribuir el material en cualquier medio o formato- y Ad aptar el documento —remezclar, transformar y crear a partir del material一 para cualquier propósito, inchso para fines comerciales, siempre que cumpla la condición de:

Atribución: Usted debe dar crédito a la obra original de manera adecuada, proporcionar un enlace a la licencia, e indicar si se han realizado cambios. Puede hacerlo en cualquier forma razonable, pero no de forma tal que sugiera que tiene el apoyo del licenciante olo recibe por el usoque hace de la obra.

Resumendelicencia - Textocompletodelalicencia 